\title{
A Development Model of Decision Support System for Purchasing of Laboratory Reagent
}

\author{
Anon Sudsai, Kittipong Sophonthummapharn, and Thaveesak Ganjanasuwan
}

\begin{abstract}
This paper studies the research in the area of analysis hierarchy process (AHP), goal programming (GP), time series analysis (TSA) and expert system (ES), which resulted in the development of the purchasing of laboratory reagent system (PLRS). This paper also introduces how a decision support system for purchasing of laboratory reagent system can be modeled, constructed, explored and explained. It is not only to promote the automatic process through a systematic framework of decision support system for purchasing of laboratory reagent, but also to increase the performance of optimize cost and to highly benefit the pioneer who uses this approach.
\end{abstract}

Index Terms-analysis hierarchy process, goal programming, time series analysis, expert system, purchasing of laboratory reagent.

\section{INTRODUCTION}

Treatments in health service place take a pathological analysis for support doctor in decision making. Information of pathological is measured the chemical, pathogen and other in the body. This results in making report of state of the body for treatment and health planning because the information are used for assign the method and planning the treatment. The implementation of edge technology equipment for pathological analysis helps reduce staffs reducing and able to analysis greater in speed and accurate. The problems in the case study include the laboratory reagents are expensive, each laboratory reagents are different how to use and maintenance, the cost of maintenance is quite too high, the loss of opportunity from demand of laboratory reagents is more increase, and the loss of opportunity from laboratory reagents are expired. These problems require management and forecasting present and future demand for use each type of laboratory reagents. The work incorporates analytic hierarchy process in choosing the best suppliers with goal programming for the purpose of purchasing the total number of each type of laboratory reagents that enables the lowest cost. Then proposed a time series analysis technique is for forecasting the demand for each type of laboratory reagents during a particular time. Finally, using a consulting/expert system for the identification of nonlinear time series analysis is for optimize cost efficiency.

It is generally accepted that decision support system for purchasing of laboratory reagent is a larger and more

Manuscript received March 14, 2011.

Suvarnabhumi Business School,Rajamangala University of Technology Suvarnabhumi,Ayutthaya, Thailand

Anon Sudsai ,wongree@gmail.com

Kittipong Sophonthummapharn,kittipong@hotmail.com

Thaveesak Ganjanasuwan ,eddy_tg@hotmail.com complex domain problem. Developers are now building enterprise-wide and global applications that must operate across corporations and continents. More and more corporations need to integrate their information systems with those of their decision maker and developers. Developing an application for these existing and emerging application domains requires powerful new methods and techniques for conceptualizing and implementing software systems.

Accordingly, a framework has been set up with adoption of the analytic hierarchy process, goal programming, time series analysis and expert system, such that a decision support system for purchasing of laboratory reagent system becomes "intelligent." The following three objectives are to develop a decision support system for purchasing of laboratory reagent system model, to develop the purchasing of laboratory reagent system, and to evaluate the developed model and system by experts and users respectively.

\section{THEORY AND RELATED RESEARCH TOPICS}

\section{A. Analytic Hierarchy Process (AHP)}

Analytic Hierarchy Process is an intuitive and efficient method for multi-criteria decision-making (MCDM) applications [1]. Mathematical model [2], the first step of AHP is to divide the decision problem into sub-problems, which are structured into hierarchy levels. The element $a_{i j}$ of the comparison matrix A represents the relative importance of choice $i$ against the choice $\mathrm{j}$, implying that the element $a_{j i}$ is the reciprocal of $a_{i j}$. Let the importance value $v$ of choice $y$ be expressed as a linear combination of the importance values for each applied criterion:

$$
\begin{array}{r}
v(y)=\sum_{j=1}^{n} w_{j} v\left(y_{j}\right), \\
a_{i j} \approx \frac{w_{i}}{w_{j}}, \\
m_{i j}=\frac{1}{m_{j i}}, \\
m_{i j}=m_{i k} m_{k j} \forall i, j, k \\
a_{i j}=\frac{w_{i}}{w_{j}} .
\end{array}
$$$$
\text { And } \quad m_{i j}=m_{i k} m_{k j} \forall i, j, k \text {. }
$$

Now let us define the matrix of weight ratios by

or, in matrix notation,

$$
W=w w^{T} .
$$

Note that Eqs. (3) and (4) hold for the matrix $W$ :

$$
w_{i j}=\frac{w_{i}}{w_{j}}=\frac{w_{i}}{w_{k}} \frac{w_{k}}{w_{j}}=w_{i k} w_{k j},
$$

Hence the matrix of weight ratios is consistent. 


\section{B. Goal Programming (GP)}

The goal programming is a branch of multi objective optimization, which in turn is a branch of multi-criteria decision analysis (MCDA), also known as multiple-criteria decision making (MCDM). [3] It is often not possible to encompass the objectives of an optimization problem within a single overriding function. A traditional approach to multiple objective problems is to assign weights to the individual objectives. In goal programming, all objectives are treated as constraints after assigning each a specific numerical goal level [4]. The objective is to minimize;

$$
z=\sum_{i=1}^{m}\left(w_{i}^{+} y_{i}^{+}+w_{i}^{-} y_{i}^{-}\right)
$$

with the goal constraints as $\sum_{i=1}^{n} c_{i j} x_{j}+y_{i}^{-}-y_{i}^{+}=g_{i} \quad \forall i$

$$
y_{i}^{-}, y_{i}^{+} \geq 0 \quad \forall i, j
$$

For example, weighting by the Euclidean norm (14) becomes

$$
\begin{array}{r}
\qquad \begin{aligned}
\sum_{j=1}^{n} c_{i j} x_{j}+\eta\left(y_{i}^{-}-y_{i}^{+}\right) & =g_{i} \quad \forall i \\
y_{i}^{-}, y_{i}^{+} & \geq 0 \quad \forall i, j \\
\eta=\left\|c_{i j}\right\| & =\left[\sum_{j=1}^{n} c_{i j}^{2}\right]^{1 / 2}
\end{aligned}
\end{array}
$$

\section{Time Series Analysis (TSA)}

The time series analysis comprises methods for analyzing time series data in order to extract meaningful statistics and other characteristics of the data. Time series forecasting is the use of a model to forecast future events based on known past events: to predict data points before they are measured. Time series are very frequently plotted via line charts [5]. This is a mathematical model generally as follows.

\section{1) Moving Average}

$$
\hat{Y}_{t+1}=\int\left(Y_{t}, Y_{t-1}, Y_{t-2}, \ldots, Y_{t-n}\right)
$$

This technique is understood easily for apply, the forecast of time series in period $\mathrm{t}+1$ ( $\hat{Y}_{t+1}$ ) will mean the sum of the actual total by the number of observation periods $(\mathrm{k})$ a model equation as follows.

$$
\hat{Y}_{t+1}=\frac{Y_{1}+Y_{t-1}+Y_{t-2}+\ldots+Y_{t-k+1}}{k}
$$

2) Trend Analysis

Trend is component of the data that show the changes in long-term that enough to be seen whether the trend of future data. Be increased or decreased. Thus, for $\mathrm{T}$ must have at least a year or more (if the monthly data) the trend line shows a straight line or curve time [6]. Trend analysis is a correlation between the trends (the value of the actual data) with the values change to $(\mathrm{t})$ the relationship of the trend $(\mathrm{T})$ with time $t$ is correlated with the form of linear or non-linear model relationships. In this case study, will use only the linear relationship. If the trend $(\mathrm{T})$ with time $(\mathrm{t})$ in a linear relationship.

\section{3) Single Exponential Smoothing}

A technique for finding the average of the non-variant data by assign the added weight to the data in each of the past was different. The model is as follows.

$$
\hat{Y}_{t+1}=\hat{Y}_{t}+\alpha\left(Y_{t}-\hat{Y}_{t}\right)
$$

4) Holt's Method or Double Exponential Smoothing

A technique for forecasting with time series data with linear trends. When the observable fact $\left(Y_{t}\right)$ will be used to calculate the expected level $\left(E_{t}\right)$ and the rate of increase or decrease the time $\left(T_{t}\right)$ with the following equation.

$$
\begin{aligned}
& \hat{Y}_{t+1}=E_{t}+n T_{t} \\
E_{t}= & \alpha Y_{t}+(1-\alpha)\left(E_{t-1}+T_{t-1}\right) \\
T_{t}= & \beta\left(E_{t}-E_{t-1}\right)+(1-\beta) T_{t-1}
\end{aligned}
$$

Assign

$$
0 \leq \alpha \leq 1 \text { and } 0 \leq \beta \leq 1
$$

Decision-makers can use the equation (16) for forecasting the future period of $n$ by $n=1,2,3, \ldots$.

5) Winter's Method or Triple Exponential Smoothing [6] This method suitable for the trend data and the influence of trend -season data. The data should not be yearly. It will not be able to separate from the influence of seasonality. The data should be in monthly, weekly, quarterly data and have at least 36 particulars or more. If the data is monthly or at least 12 particulars or more if the data is quarterly.

For this research, we propose time series analysis approach in the area of forecasting the number of each type of laboratory reagents that use in the future.

\section{An expert system (ES)}

In expert systems, knowledge about the problem in knowledge-based, is typically separate from the formal rules of logic and the solution search strategy in the fact of database and inference engine with drive the program solution via user interface. This permits more efficient incremental system development [7]. Special search strategies have been developed within the Artificial Intelligence community to reduce the typically large problem search space. Many of these are analogous to traditional operation research space. Many of these are to traditional operations research methods. Expert Systems have been successfully created in a variety of fields. Many of the systems solve diagnostic and treatment problem for limited classes of disease type in various areas of medicine [8]. Hayes-Roth, Waterman and Lenat [9] discuss 10 types of expert systems as interpretation, diagnosis, monitoring, prediction, planning, design, debugging, repair, instruction, and control.

For this research, expert systems which have been developed the decision support system for purchasing of laboratory reagent in the area of recommendation as suggestion, consult and support decision maker behavior.

\section{E. Related Research Topic}

The four related research topics of "Analysis Hierarchy Process (AHP), Goal Programming (GP), Time Series Analysis (TSA) and Expert System (ES)”. For “AHP-Based Classifier Combination” [2]. "Application of AHP and Goal Programming to Forecast Industrial Equipment for Purchasing and Identify Supplier" [6]. "A development model of knowledge expertise system for air traffic services" [11]. The optimistic idea is to carry out some distinguished features of [10], [2], [6], and [11] by 
integrated all to solving in multi-criteria to making decision. From [2] AHP investigate traditional linear combination schemes and propose a new combiner based on the AHP, a method frequently applied in mathematical psychology and multi-criteria decision making for choosing the best supplier, from [10] GP the paper focus on integrates a simulation model with a genetic algorithm heuristic and a goal programming model. The genetic algorithm technique offers a very flexible and reliable tool able to search for a solution within a global context for optimization cost for purchasing laboratory reagent, from [6] TSA take multi methodology to forecast and recheck what the method is the best solution for forecasting the usage of each type of laboratory reagent in particular time, and from [11] ES integrated many type such as interpretation, diagnosis, monitoring, prediction, planning, design, debugging, repair, instruction and control for induction knowledge from expertise to resolving the problems and suggest the optimize solution to making decision on target is performance reduce cost management.

\section{A MOdEL OF DECISION SUPPORT SYSTEM For PURCHASING OF LABORATORY REAGENT}

A model of the decision support system for purchasing of laboratory reagent can be purposely created as shown in Figure 1.

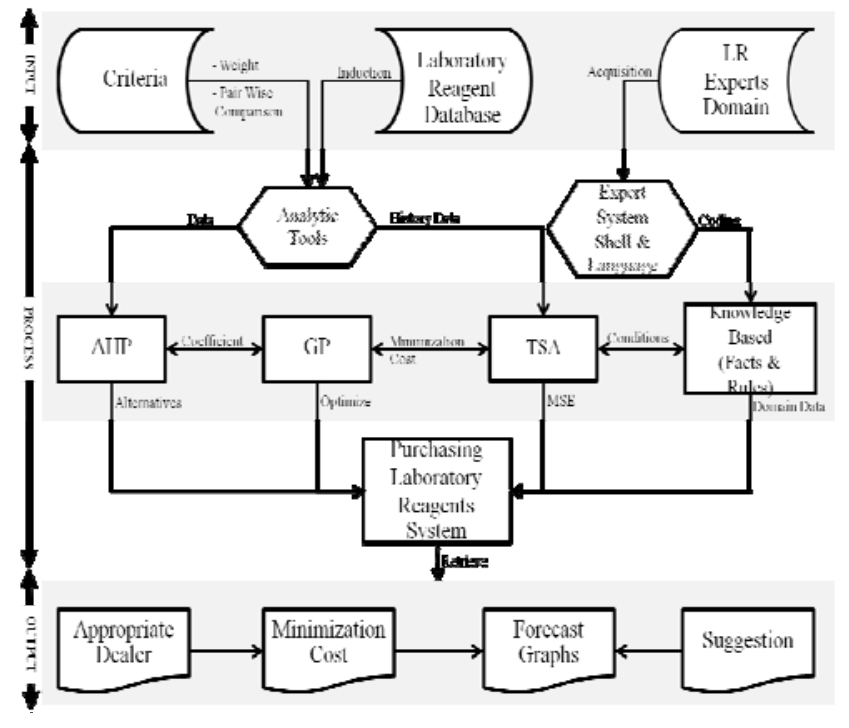

Figure 1. The model of decision support system for purchasing of laboratory reagent cycle developed by Anon Sudsai, 2010

A process models and a variety of tools, the model of decision support system for purchasing of laboratory reagent can be divided into three layers as in the following processes: Input layer, Process layer, and Output layer.

\section{F. Input Layer}

Input layer have been applied for decision support system for purchasing of laboratory reagent in a large number of different setting including;

1) LR expert domain: An expert panel was selected to determine the skills and knowledge required for the laboratory reagent domain, and to discuss whether the existing unit standards would still be fit for purpose.

2) Laboratory Reagents database: Database management system (DBMS) is to store and manage knowledge.
This worked reasonably well for induction the history data to calculate the usage of each of type of laboratory reagent.

3) Criteria: The main task is to collect, collate and analyse the obtained knowledge in the areas of laboratory reagent weight and pair wise comparison in the case of criteria which influent to decision to purchase the laboratory reagent.

\section{G. Process Layer}

The process layer design is the best described through its seven modules as explained in the following list.

1) Analytic Tool: It is an engineering discipline that involves integrating knowledge into computer systems in data induction to calculate the laboratory reagent weight and pair wise comparison to AHP.

2) Expert system shell \& language: The development of tools, call the ES shell, is specifically designed for knowledge expertise system development.

3) Knowledge-based (KB): There are three types of knowledge base entries in the system that is used to represent LR domain knowledge (LR expert domain, LR database), facts, and rules. A fact is used to directly assign values to an expression, and rules (IF-THEN) that direct the use of knowledge to solve specific problems in a particular domain.

4) Analytic Hierarchy Process (AHP): The engine used to calculate the score to create the alternative for choosing the best supplier and to send the coefficient of the equation and subsidiaries for goal programming model.

5) Goal Programming (GP): A GP used to create the relationship model to take a lowest-cost in purchases each type of laboratory reagent which coefficient from AHP.

6) Time Series Analysis (TSA): TSA used to forecast the usage of each type of laboratory reagent in the particular time in the future with five method forecasting and we decision to collect the method which the MSE smallest.

7) Purchasing Laboratory Reagent System (PLRS): A PLRS is frequently integrated with other information systems that can be develop on a knowledge platform and manage the data before display the result.

\section{H. Output Layer}

The output layer describes each part follows:

1) Appropriate Dealer: The display of the best supplier who chosen from AHP with the priorities of multicriteria under each decision criterion.

2) Minimize Cost: The display of the lowest-cost of purchasing each of type of laboratory reagent from GP process and TSA process.

3) Forecast Graphs: The display of five graphs and MSE of each methodology of forecasting from TSA process.

4) Suggestion: A knowledge expertise system works with rule-based (IF-THEN) during a suggestion the graph which the best for solution, and the system can produce an answer with a probability, although it may not be a certain one.

A model of decision support system for purchasing of 
laboratory reagent uses the development environment The user employs the consultation environment via user interface, to obtain expert knowledge and suggestion with probability.

\section{MODEL VERIFICATION AND VALIDATION}

Computer simulations and mathematical models are increasingly used to assist the process of decision making in a model of the decision support system for purchasing of laboratory reagent. The obvious concern facing both developers and users of mathematical models is the degree of confidence in the model predictions. Verification and validation $(\mathrm{V} \& \mathrm{~V})$ is designed to address this question and has become an important part of model building process.

\section{A. AHP model simulation}

Initialization: C1=Quality, C2=Payment, C3=Warranty, and C4=Delivery

1) Create pairwise comparison matrix: as shown in Table

TABLE I. WEIGHTING SCORES

\begin{tabular}{|l|c|c|c|c|}
\hline & $\mathrm{C} 1$ & $\mathrm{C} 2$ & $\mathrm{C} 3$ & $\mathrm{C} 4$ \\
\hline $\mathrm{C} 1$ & 1 & 5 & 2 & 4 \\
\hline $\mathrm{C} 2$ & $1 / 5$ & 1 & $1 / 2$ & $1 / 2$ \\
\hline $\mathrm{C} 3$ & $1 / 2$ & 2 & 1 & 2 \\
\hline $\mathrm{C} 4$ & $1 / 4$ & 2 & $1 / 2$ & 1 \\
\hline total & 1.95 & 10 & 4 & 7.5 \\
\hline
\end{tabular}

Weighting scores are $\mathrm{w} 1=0.5115, \quad \mathrm{w} 2=0.0986$, w3 $=0.2433$ and $\mathrm{w} 4=0.1466$

2) Calculate alternative suppliers

Repeat pairwise comparison each criteria with suppliers. Initialization: Sup1=Supplier 1, Sup2=Supplier 2, and Sup3 =Supplier 3; display all score as shown in Table II.

TABLE II. AHP SCORES

\begin{tabular}{|l|l|l|l|l|}
\hline & C1 & C2 & C3 & C4 \\
\hline Sup1 & 0.5714 & 0.1593 & 0.0882 & 0.0824 \\
\hline Sup2 & 0.2857 & 0.2519 & 0.6687 & 0.3151 \\
\hline Sup3 & 0.1429 & 0.5889 & 0.2431 & 0.6025 \\
\hline
\end{tabular}

3) Finding the best alternative $\mathrm{Si} \mathrm{x} \mathrm{Ai}$ "weighted sum of score"

$\mathrm{Sw}=\left[\begin{array}{llll}0.5714 & 0.1593 & 0.0882 & 0.0824 \\ 0.2857 & 0.2519 & 0.6687 & 0.3151 \\ 0.1429 & 0.5889 & 0.2431 & 0.6025\end{array}\right] \times\left[\begin{array}{l}0.5115 \\ 0.0986 \\ 0.2433 \\ 0.1466\end{array}\right]=\left[\begin{array}{l}0.3415 \\ 0.3799 \\ 0.2786\end{array}\right]$

The highest score is Supplier 2. It is the best alternative.

One of the most popular approaches to knowledge representation for expert system is to use production rules, sometimes called IF-THEN rules. It contains facts and rules relating for selecting the best suppliers, and the simulation will contain particular observations about the suppliers and criteria being examined. A typical rule in IF-THEN is compared to validate the AHP simulation as follows:

Consistency Check

$$
A w=\left[\begin{array}{cccc}
1 & 5 & 2 & 4 \\
1 / 5 & 1 & 1 / 2 & 1 / 2 \\
1 / 2 & 2 & 1 & 2 \\
1 / 4 & 2 & 1 / 2 & 1
\end{array}\right] \times\left[\begin{array}{l}
0.5115 \\
0.0986 \\
0.2433 \\
0.1466
\end{array}\right]=\left[\begin{array}{l}
2.0745 \\
0.3953 \\
0.9883 \\
0.5921
\end{array}\right]
$$

Average Ratio

$$
\frac{\frac{2.0745}{0.5115}+\frac{0.3953}{0.0986}+\frac{0.9883}{0.2433}+\frac{0.5921}{0.1466}}{4}=4.0476
$$

Consistency Index : $\frac{4.0476-4}{4-1}=0.0159$

Random Index as shown in Table III.

TABLE III. CONSISTENCY INDEX

\begin{tabular}{|c|c|c|c|c|c|}
\hline $\mathrm{N}$ & 2 & 3 & 4 & 5 & 6 \\
\hline $\mathrm{RI}$ & 0 & 0.58 & 0.90 & 1.12 & 1.24 \\
\hline \multicolumn{6}{c}{$\frac{C I}{R I}=\frac{0.0158}{0.90}=0.0176$}
\end{tabular}

if $\left(\frac{C I}{R I}<0.1\right)$ then

echo "Complete pairwise comparison"; else echo "Un-complete pairwise comparison"; echo "Please re-weighting criteria"; end

\section{B. TSA model simulation}

Initialization history data of usage then calculate and create the result graph in Moving Average as shown in Figure 2, Trend Analysis as shown in Figure 3, Single Exponential Smoothing as shown in Figure 4, Holt's method as shown in Figure 5, and Winter's method as shown in Figure 6 respectively.

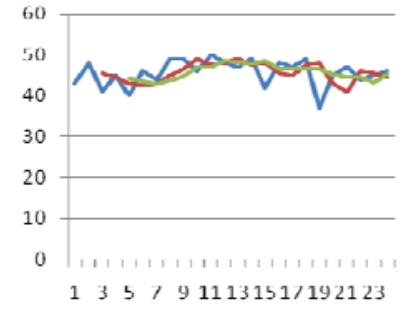

Figure 2. Moving Average

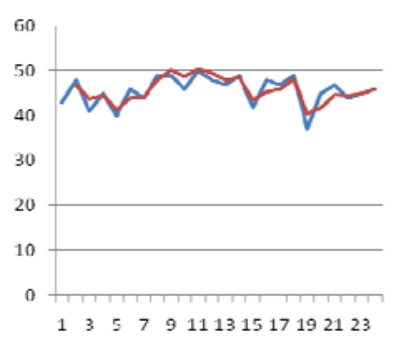

Figure 4. Single Exponential Smoothing
Figure 3. Trend Analysis

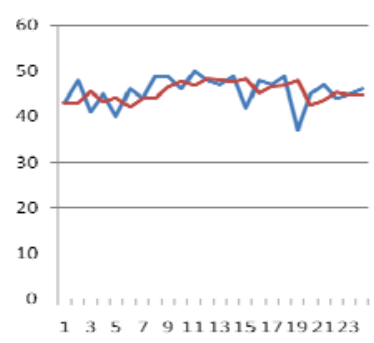

Figure 5. Holt method
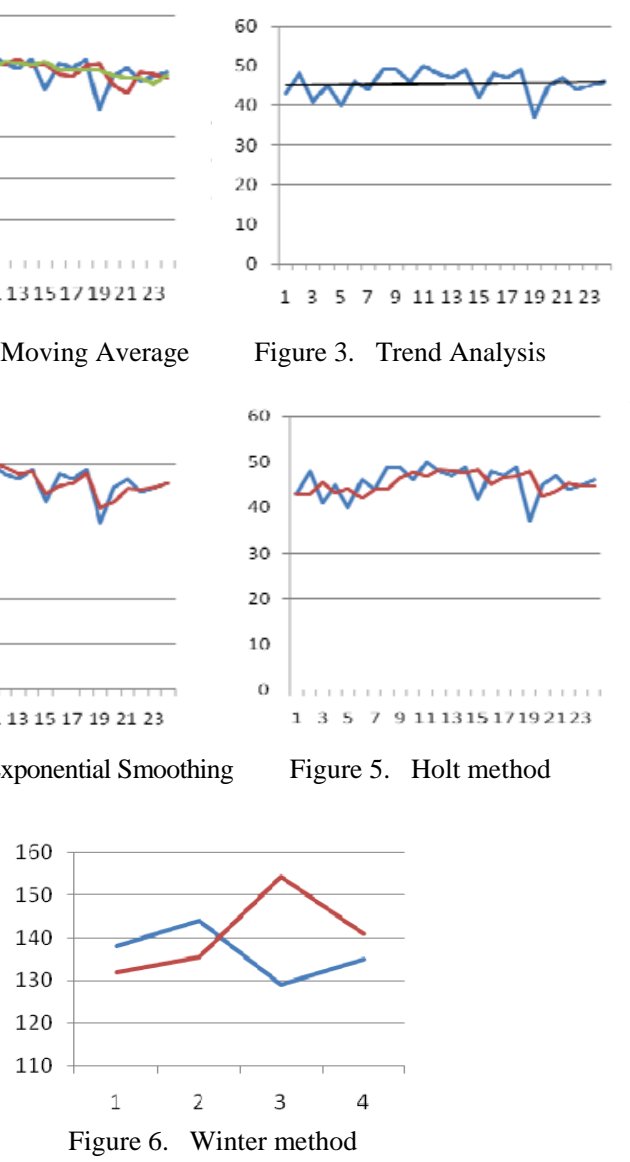

TSA forecasting plays a major role in decision making because forecasts are useful in improving the efficiency of decision-making process. This forecasting techniques to predict future demand for supplier and inventory control and accordingly take simultation decisions in expert sytem rule as follows;

String sugg_text="';

Double MSE[] $=\left\{\mathrm{MSE}_{\mathrm{MA}}, \mathrm{MSE}_{\text {Trend }}, \mathrm{MSE}_{\mathrm{Expo},}, \mathrm{MSE}_{\mathrm{Holt}}, \mathrm{MSE}_{\mathrm{Winter}}\right\}$ 
String MSE_TXT[]=\{”Moving Average"," Trend Analysis"," Exponential Smoothing”, "Holt's method", " Winter's method"\}

for $(i=0, i<=5, i++)\{$

if(min(MSE[i])) then

sugg_text= MSE_TXT[i]; end

In this case $\mathrm{MSE}_{\text {Trend }}$ is smallest, the system suggest Trend Analysis.

\section{GP model simulation}

The goal programming model presented was constructed as a practical optimization technique applied to the history matching of actual score of suppliers. The results showed that this technique is a powerful tool for optimizing the differences between the actual and predicted performance of AHP scores as shown in Table V.

\begin{tabular}{|l|c|c|c|c|}
\multicolumn{1}{c}{ TABLE IV. } \\
\hline & C1 & C2 & C3 & C4 \\
\hline Sup1 & 0.5714 & 0.1593 & 0.0882 & 0.0824 \\
\hline Sup2 & 0.2857 & 0.2519 & 0.6687 & 0.3151 \\
\hline Sup3 & 0.1429 & 0.5889 & 0.2431 & 0.6025 \\
\hline
\end{tabular}

From forecast value of trend analysis is $\mathbf{4 5 . 9 5}$

$$
z=\sum_{i=1}^{m}\left(w_{i}^{+} y_{i}^{+}+w_{i}^{-} y_{i}^{-}\right)
$$

with the goal constraints as Quality, Payment, Warranty, Delivery, and Quantity that can be shown below.

$$
\begin{gathered}
\sum_{i=1}^{n} c_{i j} x_{j}+y_{i}^{-}-y_{i}^{+}=g_{i} \quad \forall i \\
y_{i}^{-}, y_{i}^{+} \geq 0 \quad \forall i, j
\end{gathered}
$$

Replace with

1. $\mathrm{C}_{11}=0.5714, \mathrm{C}_{21}=0.1593, \mathrm{C}_{31}=0.0882, \mathrm{C}_{41}=0.5714$

2. $\mathrm{C}_{12}=0.2857, \mathrm{C}_{22}=0.1593, \mathrm{C}_{32}=0.0882, \mathrm{C}_{42}=0.5714$

3. $\mathrm{C}_{13}=0.5714, \mathrm{C}_{23}=0.1593, \mathrm{C}_{33}=0.0882, \mathrm{C}_{43}=0.5714$ $\mathrm{X}_{\mathrm{j}}=\{0,1\}$

The GP calculate the AHP scores and quantity criteria to suggest Supplier 2 is the best alternative, and then to compare the expert system simulation as follows.

\section{String}

goal[]=\{“Quality”,"Payment”,"Warranty”,"Delivery $\}$ for $(j=1, i<=4, j++)\{$

for $(i=1, i<=3, i++)\{$

$$
\operatorname{if}\left(\max \left(\mathrm{S}_{\mathrm{ji}}\right)\right) \text { then } \operatorname{goal}[\mathrm{i}] \quad \sum_{i=1}^{n} c_{i j} x_{j}+y_{i}^{-}-y_{i}^{+}=S_{j i}
$$

end $\}$ \}

\section{II-PRS DEMONSTRATION OVERVIEW}

The purpose of PLRS demonstration is to illustrate the use of decision support system for purchasing laboratory reagents as shown below.

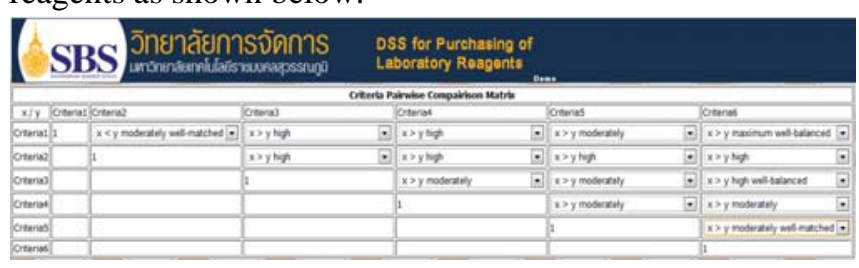

Figure 7: Criteria Pairwise Compairison Matrix

Figure 7 illustrates "Criteria Pairwise Comparison Matrix” screenshot, weighting the criteria which affect

\begin{tabular}{|c|c|c|c|c|c|}
\hline \multicolumn{6}{|c|}{ Pairwise Compairison between Supphier and Criteria1 } \\
\hline$x / y$ & Supplier1 & Supplier1 & Supplier1 & & Supplier1 \\
\hline Supplier1 & & $x>y$ moderately & $x>y$ moderately & - & $x>y$ moderately well-matched - \\
\hline Supplier1 & & 1 & $x<y$ moderately & $\nabla$ & $x<y$ moderately \\
\hline Supplier1 & & & 1 & & $x<y$ moderately \\
\hline Supplier1 & & & L & & 1 \\
\hline
\end{tabular}
decision making to know which one of criteria is the most importance and expert system check consistency index for reasonableness.

Figure 8: Pairwise comparison between supplier and each criteria

Figure 8 illustrates "Pairwise comparison between supplier and each criteria” screenshot, weighting between suppliers and each criteria to know the best alternative for selected supplier who can support purchasing factor in multi-criteria. All completed weighting and expert system check consistency index as shown below.

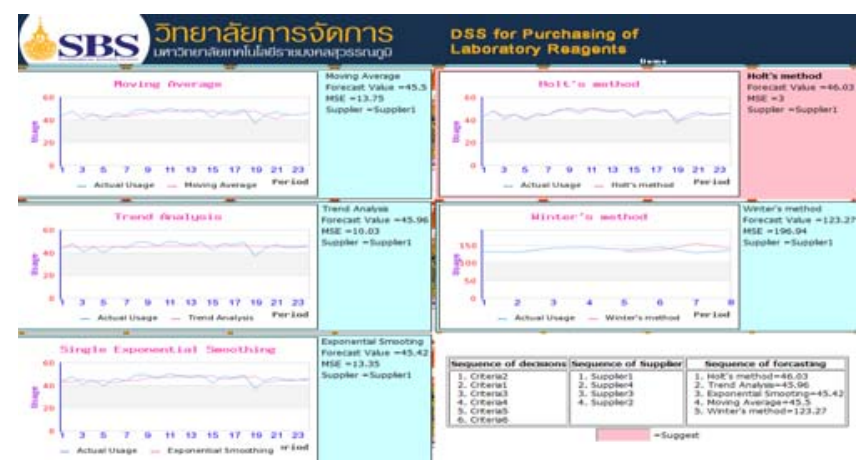

Figure 9: Result of system

Figure 9 illustrates "Result of system" screenshot, which is result of the "AHP" and "GP" in the best alternative of supplier, "TSA" in graphs and forecast value of usage in the particular time, and "ES" in suggestion the best method of forecasting.

However, if decision-maker do not want to use alternative from the system suggestion. They can discuss all information in table slot which included 1) Sequence of decisions 2) Sequence of Supplier, and 3) Sequence of forecasting. They can select the best alternative for purchase the laboratory reagents by themselves.

\section{CONCLUSION AND FUTURE WORK}

This paper introduces a purposed model of decision support system for purchasing of laboratory reagent. It consists of three crucial components: Input Layer, Process Layer and Output Layer. In the context, the model is to customize and exploit in the form of an integrated expert systems, fact and rule-based. The use of this model can create benefits including an optimization decisions, efficiency to reduce costs, capture of scarce expertise and improved decision-making processes: The systems provide rapid feedback on decision consequences, facilitate communication among decision makers, and allow rapid response to unforeseen changes in the environment, thus providing a better understanding of decision-making situation.

A proposed new model emphasizes science and technological innovation by integrating analysis hierarchy process, goal programming, time series analysis, and expert system, which resulted in the development of the purchasing of laboratory reagent system (PLRS).

This paper concludes by outlining the initial form of PLRS evaluation. The evaluation's experts resulted in a 
mean 3.75 as good satisfied. The result indicates that the new system works properly in all functions.

\section{ACKNOWLEDGMENT}

I wish to express my sincere gratitude and indebtedness to my research sponsor Suvarnabhumi Business School, Rajamangala University of Technology Suvarnabhumi and my adviser K. Sophonthummapharn, T. Ganjanasuwan and C. Yawut for his valuable guidance, advice and encouragement in doing this research.

\section{REFERENCES}

[1] Saaty, T. L., “The Analytic Hierarchy Process,” McGraw-Hill, New York, 1980.

[2] László F., “AHP-Based Classifier Combination,” Department of Informatics University of Szeged. H-6720 Szeged, 'Arpád tér 2., Hungary, 2004.

[3] F. Soudi, K. Tomsovic, "Optimal Trade-offs in Distribution Protection Design," School of Electrical Engineering and Computer Science Washington State University, Pullman, WA, 2001.
[4] Hannan, E. L., "An Assessment of Some Criticisms of Goal Programming,” Comp. Operations Research 12, 1985.

[5] Panida P., Yutthapoom W. "Analysis and make decisions of business problems using excel,” KTP Comp. \& Consult, Bangkok, Thailand, 2003.

[6] Sumasoun C., "Application of AHP and Goal Programming to Forecast Industrial Equipment for Purchasing and Identify Supplier," Industrial Engineering King Mongkut's Institute of Technology North, Bangkok, 2006.

[7] Nau, Dana S., "Expert Computer Systems”, Computer, February, 1983

[8] Buchanan, B. G. and E. H. Shortliffe, Rule-Based Expert Systems: The MYCIN Experiments of the Stanford Heuristic Programming Project. Reading, Mass: Addison-Wesley, 1984.

[9] Hayes-Roth, Frederick, D. A. Waterman, and D. B. Lenat, "An Overview of Expert Systems.” In Building Expert Systems, edited by R. HayesRoth, D.A. Waterman and D.B. Lenat. Reading, Mass: AddisonWesley, 1983.

[10] Felipe F. B.José A. S., "Multi-response simulation optimization using stochastic genetic search within a goal programming framework" Industrial Engineering and Management Systems University of Central Florida, P.O. Box 162450 Orlando, FL 32816-2450, U.S.A., 2000.

[11] Ganjanasuwan, T. Nimfuk, S., "A development model of knowledge expertise system for air traffic services", AEROTAHI, Bangkok, Thailand, 2009. 